\title{
Possibility of collision between co-orbital asteroids and the Earth
}

\author{
R.C. DOMINGOS ${ }^{1}$ and O.C. WINTER ${ }^{2,1}$ \\ ${ }^{1}$ Instituto Nacional de Pesquisas Espaciais, INPE \\ 12227-010 São José dos Campos, SP, Brazil \\ ${ }^{2}$ Grupo de Dinâmica Orbital e Planetologia, UNESP \\ Cx. Postal 205, 12516-410, Guaratinguetá, SP, Brazil \\ E-mails: rcassia@feg.unesp.br/ocwinter@feg.unesp.br
}

\begin{abstract}
Considering hypothetical co-orbital asteroids with the Earth, Tatum (1997) presented a study about the necessary radial distance in order to produce collision. He adopted an analysis considering a composition of circular two-body systems, Sun-Earth and Sun-Asteroid. He found that asteroids with radial positions between 0.9943 and $1.0057 \mathrm{AU}$ would necessarily collide with the Earth. In the present work, we show that this problem cannot be treated in this way. Adopting the circular restricted three-body problem, we verified that asteroids in the supposed region of collision with the Earth have stable horseshoe trajectories. Therefore, in the co-orbital region the dynamical system has to be studied as a restricted three-body problem since the composition of two-body problems gives a misleading result.
\end{abstract}

Mathematical subject classification: M50.

Key words: co-orbital, collision, Earth, asteroid.

\section{Introduction}

According to observations of the Spacewatch program there is a class of small asteroids (less than 50m) with similar orbits to the Earth's orbits with respect to the Sun (Rabinowitz et al., 1993). Such objects can get very close to the Earth and even collide, causing damage similar to that occurred in Tunguska (Chyba, 1993). 
On Table 1 is presented a list of asteroids that have got close to the Earth in recent years. Their distances are approximately the closest with respect to the center of the Earth. In this table are small asteroids (Chyba, 1993 and Rabinowitz et al., 1993): 1993KA2, 1991BA, 1991VG, 1993UA e 1993KA. The asteroid 1991VG has similar orbits to the Earth's, with semi-major axis 1.04 AU and eccentricity 0.067 . Since such small asteroids have similar orbits to the Earth, it is relevant to find out the orbital characteristics that would contribute or not to produce collision with the Earth.

According to Farinella et al. (1996) the significant dynamical mechanisms that may affect the orbit Earth-crossing asteroids orbits depend on time span of the effect of some secular resonances caused by Mars perturbations. This resonant perturbations on a long time span can make the asteroid to collide with the Earth. Wiegert et al. (1998) studied the orbital evolution of a near-Earth asteroid in 1:1 mean motion resonance with the Earth. It also crosses the orbits of Venus and Mars. From this study has been shown that the behavior of the horseshoe orbit is primarily under the influence of the Sun and Earth, but its long term behavior and stability are strongly affected by terrestrial (Venus and Mars) and Jovian planets. Namouni (1999) and Namouni \& Murray (2000) studied the three-body problem when co-orbiting objects can experience close encounters. They showed that considering the case of high inclination and high eccentricity there are secular mechanisms that protect asteroids against collisions with the Earth. This asteroids can be temporarily captured in co-orbital configurations or in orbits with few revolutions and escape (Christou, 2000 and Morais \& Morbidelli, 2002).

Considering hypothetical co-orbital asteroids with the Earth, Tatum (1997) presented a study about the necessary radial distance in order to produce collision. He adopted an analysis considering a composition of circular two-body systems, Sun-Earth and Sun-Asteroid. He concluded that hypothetical co-orbital asteroids with the Earth, might collide with the planet, provided that the radial distance asteroid-Earth, is the range 0.9943 and $1.0057 \mathrm{AU}$.

The model adopted by Tatum (1997) and his approximations give a misleading result.

According to the references presented, this asteroids are under planetary per- 


\begin{tabular}{|l|l|c|}
\hline Designation & \multicolumn{1}{|c|}{ Date } & $\begin{array}{c}\text { Distance } \\
\left(10^{3} \mathrm{~km}\right)\end{array}$ \\
\hline \hline 2003 SQ222 & 2003 Sept. 27 & 84 \\
1994 XM1 & 1994 Dec. 9 & 108 \\
2002 XV90 & 2002 Dec. 11 & 118 \\
2002 MN & 2002 June 14 & 120 \\
1993 KA2 & 1993 May 20 & 148 \\
2003 XJ7 & 2003 Dec. 6 & 148 \\
2003 SW130 & 2003 Sept. 19 & 162 \\
1994 ES1 & 1994 Mar. 15 & 169 \\
1991 BA & 1991 Jan. 18 & 171 \\
2003 UM3 & 2003 Dec. 12 & 281 \\
2001 BA16* & 2001 Jan. 15 & 307 \\
2003 HW10 & 2003 Apr. 29 & 331 \\
2002 GQ & 2002 Mar. 31 & 422 \\
1995 FF** & 1995 Mar. 27 & 433 \\
1996 JA1 & 1996 Mar. 19 & 454 \\
2003 XV & 2003 Dec. 6 & 458 \\
1991 VG & 1991 Dec. 5 & 458 \\
2002 EM7 & 2002 Mar. 8 & 466 \\
2002 CB26 & 2002 Feb. 8 & 480 \\
2003 LW2 & 2003 June 1 & 496 \\
1994 WR12 & 1994 Nov. 24 & 720 \\
1995 UB & 1995 Oct. 17 & 750 \\
1993 UA & 1993 Oct. 18 & 1,000 \\
1994 GV & 1994 Apr. 12 & 1,030 \\
1993 KA & 1993 May 17 & 1,060 \\
1997 UA11 & 1997 Oct. 26 & 1,060 \\
1997 CD17 & 1997 Feb. 9 & 1,110 \\
\hline
\end{tabular}

Table 1 - Some asteroids that have got close to the Earth in recent years. The distances are as given on January, 2004 by the Minor Planet Center Database available at http://cfa-WWW.harvard.edu/iau/NEO/TheNEOPage.html. To have an idea of how close are these distances, bear in mind that the Earth-Moon distance is about $384,400 \mathrm{~km}$. *The closest approach to the Moon was $79,287 \mathrm{~km}$. **The closest approach to the Moon was $194,447 \mathrm{~km}$. 
turbations in the solar System.

In the present work, the main goal is to demonstrate that asteroids in the supposed region proposed by Tatum (1997) have stable trajectories. Adopting the circular restricted three-body problem, neglecting all other perturbations, we verified that these orbits are of tadpole or horseshoe type.

In the following section we summarize the work of Tatum (1997). In section 3 we present a brief review of the theory of co-orbital motion. Our numerical simulations are shown in section 4 . The conclusion is given in the last section.

\section{Tatum's results}

Following we summarize the work of Tatum (1997). Considering only the system Earth-Moon and inserting a third body, the gravitational attraction of the Earth can be relevant in the collisional process of NEOs with the Earth. The terrestrial gravitational force will depend on the impact parameter and the relative velocity of the third body. The gravitational attraction of the Earth can implicate in a cross-section larger than the area of the maximum geometrical circle formed by Earth, called effective cross-section. Therefore, the asteroid could be pulled in from an area that may be considerably larger than Earth's geometrical cross section.

In Figure 1 is presented a diagram representing the orbit of an asteroid (A) and the Earth's orbits (E). Both orbits are assumed to be planar and circular. In the top diagram the distances between the orbits is large enough to avoid collision between the asteroid and the Earth. The asteroid does not suffer significant gravitational influence of the Earth. In the bottom diagram the distance is smaller and the effective cross-section is much larger than Earth's geometrical cross section. The asteroid collides with the Earth.

In order to determine the critical radial distance between the asteroid and the Earth, it is considered the intensity of the Earth's velocity in the inertial frame, $V_{\oplus}$, the Earth's orbital radius, $r_{\oplus}$, the asteroid's orbital radius, $r_{\oplus}+\delta r_{\oplus}$, and the intensity of the asteroid's velocity in the inertial frame, $V_{\oplus}+\delta V_{\oplus}$. In this case the symbols $\delta r_{\oplus}$ and $\delta V_{\oplus}$ are the impact parameter and the asteroid's relative velocity.

Considering the circular two-body problem Earth-asteroid, energy and angular 


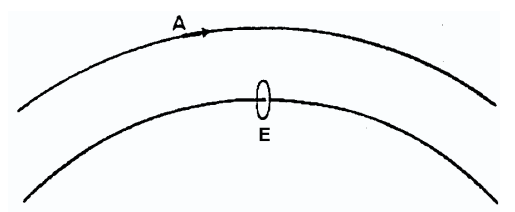

(a)

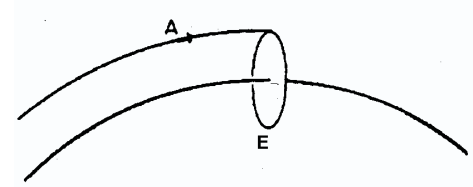

(b)

Figure 1 - Diagram representing the orbit of an asteroid (A) and the Earth's orbits (E). In figure (a) the distances between the orbits is large enough to avoid collision between the asteroid and the Earth. The asteroid does not suffer significant gravitational influence of the Earth. In figure (b) the distance is smaller and the effective cross-section is much larger than Earth's geometrical cross section. The asteroid collides with The Earth. (adapted from Tatum's paper).

moment are conserved, so

$$
v_{0}^{2}=v_{p}^{2}-\frac{2 G M_{\oplus}}{q}
$$

and

$$
v_{0} \delta r=v_{p} q
$$

where $G, M_{\oplus}, q, v_{0}$ and $v_{p}$ are gravitational constant, Earth's mass, perigee, initial and perigee velocities of the asteroid, respectively. From (1) and (2) can be deduced the following equation

$$
\frac{\delta r^{2}}{q^{2}}=1+\frac{2 G M_{\oplus}}{q v_{0}^{2}}
$$

Considering that $v_{0}=\delta V_{\oplus}$ and replace $q$ by Earth's radius, $R_{\oplus}$. In this way, the equation (3) become

$$
\delta r=R_{\oplus}\left[1+\left(\frac{V_{e}}{\delta V_{\oplus}}\right)^{2}\right]^{\frac{1}{2}}
$$


where $V_{e}$ is the escape velocity from the surface of the Earth. This is the expression for radius of the effective cross-section (or impact parameter).

Now it is considered the circular two-body problems Sun-Earth and Earthasteroid. The ratio between the orbital periods of the Earth $T_{\oplus}$ and of the asteroid $T_{A}$ is given by

$$
\frac{T_{\oplus}}{T_{A}}=\left(\frac{r_{\oplus}}{r_{\oplus}+\delta r_{\oplus}}\right)\left(\frac{V_{\oplus}}{V_{\oplus}+\delta V_{\oplus}}\right)
$$

Using Kepler's third law and equation (1), the asteroid's relative velocity is approximately given by

$$
\delta V_{\oplus}=-V_{\oplus}\left(\frac{\delta r_{\oplus}}{2 r_{\oplus}}\right)
$$

Using equations (6) and (4) one can find

$$
\left(\delta r_{\oplus}\right)^{4}-R_{\oplus}^{2}\left(\delta r_{\oplus}\right)^{2}-\left(\frac{8 G M_{\oplus} R_{\oplus} r_{\oplus}^{2}}{V_{\oplus}^{2}}\right)^{1 / 4}=0
$$

From this equation Tatum (1997) determined the minimum $\delta r_{\oplus}$ for an asteroid collide with the Earth as being 0.0057 AU (about 2,2 times the Earth - Moon distance). Therefore, from such result, asteroids with small eccentricities and semi-major axis between 0.9943 and $1.0057 \mathrm{AU}$ would collide with the Earth in the first encounter.

\section{Coorbital motion theory}

For more than two centuries it is well known that the circular restricted three-body problem presents five equilibrium points. These points are called Lagrangian equilibrium points $L_{1}, L_{2}, L_{3}, L_{4}$ and $L_{5}$. It is also known that stable orbits can evolve in the co-orbital region, oscillating around some of the Lagrangian points (see for instance Roy, 1988; Petit \& Hénon, 1986; Dermott \& Murray, 1981). The stable orbits can be of tadpole or horseshoe type. Trajectories librating around $L_{4}, L_{3}$ and $L_{5}$ are called horseshoe orbits. Horseshoe orbits are so named because of their shape in a reference frame that co-rotates with their accompanying planet. Tadpole trajectories are librating about the $L_{4}$ or $L_{5}$ Lagrangian points. These orbits are in 1:1 resonance with their accompanying 
planet. In Figure 2 is presented a diagram indicating the lagrangian points and the regions where the stable co-orbital trajectories can happen.

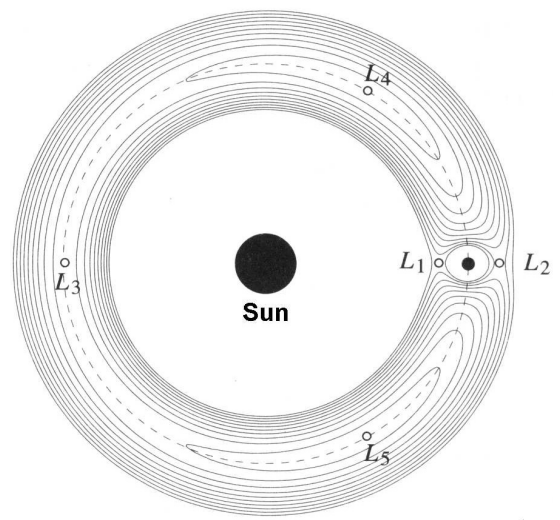

Figure 2 - Diagram indicating the lagrangian points and the regions where the stable co-orbital trajectories can happen. The horseshoe and tadpole orbits are viewed in the rotating frame. Horseshoe orbits encompass the $L_{4}, L_{3}$ and $L_{5}$ points. Tadpole orbits oscillates about the $L_{4}$ or $L_{5}$ Lagrangian points. (adapted from Murray \& Dermott, 1999).

Dermott \& Murray (1981) developed an analytical approach in order to estimate the width of the stable co-orbital region, which is expressed by $\delta r \sim\left(m_{2} / m_{1}\right)^{1 / 3}$, where $m_{1}$ and $m_{2}$ are the masses of the central body and the secondary body. In the Sun-Earth case we have $\delta r \sim 0.01443$.

\section{Numerical simulations}

In this section we present numerical results of simulations adopting the circular restricted three-body problem, Sun-Earth-Asteroid. The initial conditions of the asteroid are such that it is in a co-orbital circular orbit with the Earth, with orbital radius between $0.960,0.970,0.981,0,985,0.990$ and $0.994 \mathrm{AU}$. The values of semi-major axis cover a representative part of the analyzed region.

Figure 3 shows the trajectories from the numerical simulations in polar coordinates, $r$ and $\theta$, in a frame rotating with the Earth. From the obtained result note that there are four types of orbital evolutions of the asteroid: temporary capture around the Earth (or collision with the Earth), scattering of the orbit, formation 
of waves and stable horseshoe or tadpole orbits.

Note that the trajectories in the range $0.9943<r<1.0057 \mathrm{AU}$, believed to be trajectories of collision with the Earth (previous section), are in fact stable horseshoe or even tadpole orbits. The actual minimum approximation one of these trajectories can get from the Earth is more than $10^{\circ}$, without any chance of collision. This two bodies "repel" each other azimuthally and never come in close proximity. In fact, the width of the stable co-orbital region is delimited at least in the range $0.990 \leq r \leq 1.010$ AU. Eventually, a collision between an asteroid and the Earth can occur for trajectories just outside of the border of the stable region (see in Figure 3 the case of the trajectory with initial $r \approx 0.98 \mathrm{AU}$ ). That is due to the chaotic behavior of the region limiting the stable co-orbital region.

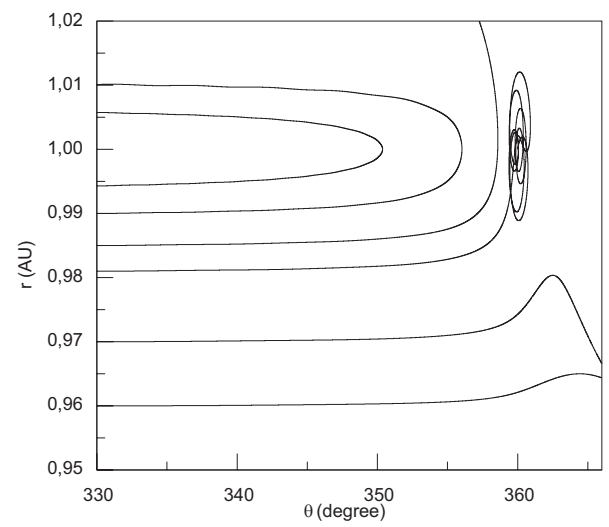

Figure 3 - Numerical simulations of the co-orbital region considering the circular restricted three-body problem, Sun-Earth-asteroid. The values are given in polar coordinates, $r$ and $\theta$, in the rotating frame. The Earth is located at $r=1.00 \mathrm{AU}$ and $\theta=0^{\circ}$.

\section{Conclusion}

The main contribution of this work is that adopting the circular restricted threebody problem, as a first approximation, we confirm that asteroids in the supposed region of collision with the Earth have stable horseshoe/tadpole trajectories. Therefore, in the co-orbital region the dynamical system has to be studied as a restricted three-body problem since the composition of two-body problems gives 
a misleading result. It is important to bear in mind that in our study we neglected all other perturbations. According with the references mentioned above, in a more realistic model other perturbations must not be neglected.

\section{Acknowledgements}

This work was funded by CNPq, Capes and Fapesp.

\section{REFERENCES}

[1] A.A. Christou, A Numerical Survey of Transient Co-orbitais of the Terrestrial Planets. Icarus, 144 (2000), pp. 1-20.

[2] C.F. Chyba, Explosions of Small Spacewatch Objects in the Earth's Atmosphere. Nature, 363 (1993), pp. 701-703.

[3] S.F. Dermott and C. D. Murray, The Dynamics of Tadpole and Horseshoe Orbits. I. Theory. Icarus, 48 (1981), pp. 1-11.

[4] C.D. Murray and S.F. Dermott, " Solar System Dynamics ”, Cambridge University Press. Cambridge, England, (1999).

[5] R.C. Domingos, “Atuação da Lua no Processo Colisional de Corpos Celestes com a Terra”, Master Thesis, UNESP, Guaratinguetá, Brazil, (2000).

[6] P. Michel, P. Farinella and Ch. Froeschlé, The Orbital Evolution of the Asteroids Eros and Implications for Collision with the Earth. Nature, 380 (1996), pp. 689-691.

[7] M.H.M. Morais and A. Morbidelli, The Population of Near-Earth Asteroids in Coorbital Motion with the Earth. Icarus, 160 (2002), pp. 1-9.

[8] F. Namouni, Secular Interactions of Coorbiting Objects. Icarus, 137 (1999), pp. 293-314.

[9] F. Namouni and C.D. Murray, The Effect of Eccentricity and Inclination on the Motion Near the Lagrangian Points $L_{4}$ and $L_{5}$. Celestial Mechanics and Dynamical Astronomy, 76 (2000), pp. 131-138.

[10] J.M. Petit and M. Hénon, Satellite Encounters. Icarus, 66 (1986), pp. 536-555.

[11] D.L. Rabinowitz, T. Gehrels, J.V. Scotti, R.S. Mcmillan, M.L. Perry, W. Wisniewski, S.M. Larson, E.S. Howell and B.E.A. Mueller, Evidence for a Near-Earth Asteroid Belt. Nature, 363 (1993), pp. 704-706.

[12] A.E. Roy, “ Orbital Motion”, Adam Hilger, Bristol, (1988).

[13] J.B. Tatum, The Capture Cross-Section of Earth for Errant Asteroids. Journal of the Royal Astronomical Society of Canada, 913 (1997), pp. 276-278.

[14] P.A. Wiegert, K.A. Innanen and S. Mikkola, The Orbital Evolution of Near-Earth Asteroid 3753. The Astronomical Journal, 115 (1998), pp. 2604-2613. 